\title{
3 Research Square \\ Barriers and Facilitators on the HIV Care \\ Continuum: a Scoping Review Protocol
}

\section{Gwang Suk Kim}

Yonsei University College of Nursing

\section{Youngin Kim}

Yonsei University College of Nursing

\section{Minkyung Park}

National Police Hospital

\section{SangA Lee}

University of Massachusetts Boston - College of Nursing and Health Sciences

\section{Youngjin Lee}

Yonsei University College of Nursing

Mi-So Shim ( $D$ msshim@yuhs.ac)

Yonsei University College of Nursing https://orcid.org/0000-0001-9948-0662

\section{Protocol}

Keywords: HIV, care continuum, barriers and facilitators, scoping review

Posted Date: February 9th, 2021

DOI: https://doi.org/10.21203/rs.3.rs-174330/v1

License: (c) (1) This work is licensed under a Creative Commons Attribution 4.0 International License. Read Full License 


\section{Abstract}

Background: As the life expectancy of people living with HIV (PLHIV) increases with the advancements in antiretroviral treatment, the continuity of long-term therapy and health care for PLHIV has gained more importance. However, the estimated proportion of PLHIV who have access to treatment or who are virally suppressed is unsatisfactory. Therefore, it is necessary to build strategies to improve treatment continuity by identifying the barriers and facilitators that affect the HIV care continuum. To enable this, we will conduct a scoping review to explore the barriers and facilitators on the care continuum for PLHIV.

Methods: Based on the scoping review framework, the research questions will identify the factors affecting the care continuum of PLHIV, supported by a literature search we will conduct on the databases of MEDLINE, EBSCO Cumulative Index to Nursing and Allied Health Literature (CINAHL), Embase, and Cochrane library. Additionally, we will review guidelines and policies on HIV care published by organizations related to HIV infection to ascertain the barriers and facilitators in the continuity of care. Four researchers will screen articles for inclusion and subsequently build a charting form and collate the data to provide results.

Discussion: The results of this scoping review will provide comprehensive evidence for influencing factors to be considered in the care continuum of PLHIV. Importantly, the results will provide insight for health care providers and researchers to develop interventions and research the continuity in caring for PLHIV.

\section{Background}

The number of people living with HIV (PLHIV) has increased globally, estimated at 38 million, as of 2019 [1]. This increase, attributable to the number of people accessing treatment, is due to the improved effectiveness of antiretroviral therapy (ART) and reduced emotional burden on taking drugs [2]. In countries such as Korea, where PLHIV can easily receive ART, a 20-year-old person diagnosed with HIV receiving continuous treatment reportedly lived into their early 70s [3]. Thus, it is necessary to ensure the continuity of long-term treatment and health care for patients with HIV, similar to patients with other chronic diseases.

Several phases of HIV treatment have been studied: (1) from the first diagnosis to the treatment initiation $[4,5],(2)$ to a few months of ART to verify its effectiveness for PLHIV $[4,6],(3)$ to treatment retention, and (4) a long-term shift to improving health behaviors for those with stable HIV status [7, 8]. In 2015, as a global agreement on these phases, the Joint United Nations Programme on HIV/AIDS (UNAIDS) proclaimed the target of achieving " $90-90-90$ ' by $2020 ; 90 \%$ of PLHIV diagnosed, $90 \%$ of those diagnosed antiretrovirally treated, and $90 \%$ of those treated virally suppressed [9]. This led to the consensus that, as a chronic disease, it was more important to maintain the HIV care continuum-from the first diagnosis to long-term health care - to emphasize the specific periods in HIV care. 
Despite the 90-90-90 indicator, among all patients with HIV, the estimated proportion of patients who accessed treatment is about $67 \%$, and patients who were virally suppressed is $59 \%$ [1]. These proportions indicate a current need for interventions to maintain the HIV care continuum. For developing such interventions, it is necessary to synthesize barriers and facilitators identified throughout the HIV care continuum.

The HIV care continuum emphasizes maintaining the health of PLHIV and preventing transmission through adherence to treatment $[10,11]$. Systematic reviews of the barriers and facilitators of the HIV care continuum, reported in the previous studies, were limited to patients with HIV in specific populations [12, 13], or only included a specified time point of the HIV care continuum [14]. Additionally, in a systematic review, there is a limitation in that neither quantitative nor qualitative studies are comprehensively analyzed and integrated [15]. To overcome these limitations, we intend to conduct this scoping review to explore the influencing factors throughout the care continuum for PLHIV. Scoping review is useful when the knowledge on topic has not been comprehensively reviewed, or the subjects of exploration has a complex and heterogeneous nature [16]. Through this review, we aim to provide comprehensive knowledge about barriers and facilitators that should be considered in the caring of people with HIV in clinical fields.

\section{Methods}

We developed this protocol based on the scoping review framework proposed by Arksey and O'Malley (2005) and as recommended by Levac and colleagues [17-19]. In addition, we plan to follow the Preferred Reporting Items for Systematic reviews and Meta-Analyses extension for scoping reviews (PRISMA-ScR) checklist to perform the scoping review [20]. The detailed research process and content are as follows:

\section{Framework stage 1: Identifying the research question}

The research questions in this review will help to identify the factors affecting the care continuum of PLHIV. The specific research questions are as follows:

1) What are the barriers that affect the care continuum of PLHIV identified in the existing evidence?

2) What are the facilitators that affect the care continuum of PLHIV identified in the existing evidence?

3) Are each identified barriers and/or facilitators located at any particular period or the entire period of the HIV care continuum?

\section{Framework stage 2: Identifying related studies}

As a research team, with the assistance of a librarian, we have developed a comprehensive search strategy (supplemental materials 1). We plan to conduct a literature search from the database of MEDLINE, EBSCO, Cumulative Index to Nursing and Allied Health Literature (CINAHL), Embase, and Cochrane library that will include peer-reviewed articles published in English or Korean between 2013 and 
2020. The search period was set to begin from 2013 because the guidelines to start ART changed from when the number of CD4 immune cells fell below a certain level to immediately after the diagnosis of HIV infection $[21,22]$.

In addition, we will review guidelines and policies on HIV care published by organizations related to HIV infection such as the World Health Organization (WHO), International Association of Providers of AIDS Care (IAPAC), Centers for Disease Control and Prevention (CDC), The U.S. Department of Health and Human Services (HHS), and Korean Centers for Disease Control and Prevention (KCDC). These will be useful to review the methodological quality of the studies included in the scoping review.

\section{Framework stage 3: Study selection}

First, the results retrieved from each database will exclude duplicate articles using the Endnote X9.2 program. Three researchers will screen titles and abstracts to exclude articles that do not meet eligibility criteria. Second, a review of full-text articles will determine which to include in the final analysis. Last, we will report this process using the PRISMA flow diagram (Fig. 1). To ensure systematic processing, we will conduct adequate discussions and training prior to screening and selection, and report the progress through weekly research meetings and discussion of results.

\section{Eligibility criteria}

For this review, the inclusion criteria are articles on (1) people with HIV over the age of 19 and (2) outcomes (barriers and facilitators) that present the factors affecting HIV care. Concurrently, the exclusion criteria are articles on (1) children and adolescents, (2) HIV prevention in non-infected persons, (3) only for the population in a specific context or situation (e.g., transgender people, inmates, pregnant women, refugees, etc.), (4) methodological research, such as scale development or program development, (5) trial or review protocol, and (6) expert opinions and insights articles that do not include original data.

\section{Framework stage 4: Charting the data}

The research team will build a charting form through discussions, including characteristics of the screened articles and outcomes. Table 1 shows the expected contents to be included in the charting form. Prior to charting, researchers will be provided sufficient training on the methods of data abstraction. Subsequently, each researcher will perform data abstraction of five randomly selected articles and discuss the results, modifying the charting form if necessary. If the need arises for a collaborative discussion on specific aspects during the charting process, each researcher will record and present these at weekly review meetings.

\section{Framework stage 5: Collating, summarizing, and reporting the results}

The barriers and facilitators will be classified according to the care continuum presented in each study, followed by frequency analysis. Through this process, the research team can identify barriers and facilitators that show (1) high frequency and (2) conflicting results from the research thus far. 
Furthermore, the synthesized results will enable the research team to present the framework on barriers and facilitators in the HIV care continuum and propose implications for future research, practice, and policy.

\section{Framework stage 6: Consultation exercise}

This review will not include the consultation exercise stage.

\section{Quality assessment}

Levac and colleagues suggested collecting evidence from stakeholder consultation and using a critical appraisal tool to ensure that the scoping review meets the standard quality [19]. To identify the evidence from stakeholder consultation, we will gather experts' opinion from the PLHIV, who are consumers of these exploration topic, and nurses and physicians who provide health services across the care continuum. List of barriers and facilitators configured through the five-step process will be provided to the experts in order to evaluate the validity of each item of list based on their own experience and knowledge. The quality of the selected studies will be evaluated using the mixed method appraisal tool (MMAT) version 2011 [23]. Moreover, at a weekly review meeting, we will discuss the quality of these selected studies and whether to exclude them from the analysis.

\section{Discussion}

This scoping review aims to identify barriers and facilitators that affect improving the continuity of care for PLHIV. The purpose of this scoping review is to facilitate a comprehensive analysis and integration of existing evidence on the specific details of barriers and facilitators affecting the HIV care continuum. For this analysis, we intend to compare the barriers and facilitators found in academic articles and guidelines, and identify the similarities and differences between the two types of literature. The results to be confirmed through this review will provide the content to constitute strategies designed to reduce barriers and improve facilitators in the continuity of care by health care providers in clinical fields. In addition, these results can also be useful as evidence for policies and guidelines for PLHIV, and provide insight into future research topics and directions to reduce knowledge gap regarding the care continuum of PLHIV.

One of the limitations of our review protocol is that since we have included only English and Korean articles in the analysis, selection bias may occur. To mitigate this limitation, we will review the guidelines of organizations associated with HIV. Additional limitations that may arise in the process of this study will be described in the full text scoping review.

\section{List Of Abbreviations}

ART: Antiretroviral Therapy 
HIV: Human immunodeficiency virus

PLHIV: People living with HIV

\section{Declarations}

\section{Ethics approval and consent to participate:}

Not applicable

\section{Consent for publication:}

Not applicable

\section{Availability of data and materials:}

All data generated or analyzed during this study will be included in the published scoping review article.

\section{Competing interests:}

The authors declare that they have no competing interests.

\section{Funding:}

This work was supported by the National Research Foundation of Korea (NRF) grant funded by the Korea government (MSIT) (No. 2020R1A2C101081711) and the Brain Korea 21 FOUR Project funded by National Research Foundation (NRF) of Korea, Yonsei University College of Nursing.

\section{Authors' contributions:}

G.S.K. was the project facilitator. M.S. and G.S.K. were main authors. M.S., M.K.P., Y.I.K., and S.A.L. reviewed selected studies primarily. G.S.K., M.S., Y.I.K., M.K.P., S.A.L. and Y.J.L all contributed towards the background, research design, and discussion. All authors read and approved the final manuscript.

\section{Acknowledgements:}

The authors would like to thank Na Won Kim, Librarian, Yonsei University Medical Library.

\section{References}

1. The Joint United Nations Programme on HIV/AIDS (UNAIDS). Global HIV \& AIDS statistics - 2020 fact sheet. https://www.unaids.org/sites/default/files/media_asset/UNAIDS_FactSheet_en.pdf Accessed 6 Dec 2020

2. Vitoria M, Rangaraj A, Ford N, Doherty M. Current and future priorities for the development of optimal HIV drugs. Curr Opin HIVAIDS. 2019;14(2):143-149. 
3. Marcus JL, Chao CR, Leyden WA, Xu L, Quesenberry Jr CP, Klein DB, et al. Narrowing the gap in life expectancy between HIV-infected and HIV-uninfected individuals with access to care. J Acquir Immune Defic Syndr. 2016;73(1):39-46.

4. Aaron E, Alvare T, Gracely EJ, Riviello R, Althoff A. Predictors of linkage to care for newly diagnosed HIV-positive adults. West J Emerg Med. 2015;16(4):535-542. doi: 10.5811/westjem.2015.4.25345

5. Shim, M, Kim S, Park CG. Factors influencing the initiation of treatment after the diagnosis of Korean patients with HIV. J Korean Acad Community Health Nurs. 2018;29(3):279-289. doi: 10.12799/jka chn.2018.29.3.279

6. Delpech, V, Brown, AE, Croxford S, Chau C, Polavarapu V, Cooper N, et al. Quality of HIV care in the United Kingdom: Key indicators for the first 12 months from HIV diagnosis. HIV Med. 2013;Suppl 3:19-24. doi: 10.1111/hiv.12070

7. Pecoraro A, Royer-Malvestuto C, Rosenwasser B, Moore K, Howell A, Ma M, et al. Factors contributing to dropping out from and returning to HIV treatment in an inner city primary care HIV clinic in the United States. AIDS Care. 2013;25(11):1399-1406. doi: 10.1080/09540121.2013.772273

8. Puskas CM, Kaida A, Miller CL, Zhang W, Yip B, Pick N, et al. The adherence gap: a longitudinal examination of men's women's antiretroviral therapy adherence in British Columbia, 2000-2014. AIDS. 2017;31(6):827-833. doi: 10.1097/QAD.0000000000001408

9. The Joint United Nations Programme on HIV/AIDS (UNAIDS). 90-90-90: an ambitious treatment target to help end the AIDS epidemic. Geneva: UNAIDS; 2014. Accessed 10 Dec 2020.

10. Li Z, Purcell DW, Sansom SL, Hayes, Hall HI. Vital signs: HIV transmission along the continuum of care - United States, 2016. MMWR Morb Mortal Wkly Rep. 2019;68(11):267-272. doi: 10.15585/mmwr.mm6811e1

11. Williams EC, McGinnis KA, Edelman EJ, Matson TE, Gordon AJ, Marshall BDL, et al. Level of alcohol use associated with HIV care continuum targets in national U.S. sample of person living with HIV receiving healthcare. AIDS and Behav. 2019;23(1):140-151. doi: 10.1007/s10461-018-2210

12. Jones $C$, Ritchwood TD, Taggart T. Barriers and facilitators to the successful transition of adolescents living with HIV from pediatric to adult care in low and middle-income countries: a systematic review and policy analysis. AIDS and Behav. 2019;23(9):2498-2513. doi: 10.1007/s10461-019-02621-6

13. Geter A, Sutton MY, Hubbard McCree D. Social and structural determinants of HIV treatment and care among black women living with HIV infection: a systematic review: 2005-2016. AIDS Care. 2018;30(4):409-416. doi: 10.1080/09540121.2018.1426827

14. Kelly N, Maokola W, Mudasiru O, McCoy SI. Interventions to improve linkage to HIV care in the era of "Treat All" in sub-Saharan Africa: a systematic review. Curr. HIV/AIDS Rep. 2019;16(4):292-303. doi: 10.1007/s11904-019-00451-8

15. Hall BJ, Sou KL, Beanland R, Lacky M, Tso LS, Ma Q, et al. Barriers and facilitators to interventions improving retention in HIV care: a qualitative evidence meta-synthesis. AIDS and Behav. 2017;21(6):1755-1767. doi:10.1007/s10461-016-1537-0 
16. Peters MDJ, Godfrey CM, Khalil H, Mclnerney P, Parker D, Soares CB. Guidance for conducting systematic scoping reviews. International Journal of Evidence-Based Healthcare. 2015;13(3):141146. doi: $10.1097 /$ XEB0000000000000050

17. Arksey H, O'Malley L. Scoping studies: towards a methodological framework. Int J Soc Res Method. 2005;8(1):19-32.

18. Seo H, Kim S. What is scoping review? Journal of Health Technology Assessment. 2018;6(1):16-21.

19. Levac D, Colquhoun H, O'Brien KK. Scoping studies: advancing the methodology. Implementation Sci. 2010;5:69. doi:10.1186/1748-5908-5-69

20. Tricco AC, Lillie E, Zarin W, O'Brien KK, Colquhoun H, Levac D, et al. PRISMA Extension for Scoping Reviews (PRISMAScR): Checklist and Explanation. Ann Intern Med. 2018;169(7):467-473. doi: 10.7326/M18-0850.

21. World Health Organization (WHO). Global update on HIV treatment 2013: results, impact and opportunities. Geneva: WHO; 2013. Accessed 10 Dec 2020.

22. The Korean Society for AIDS. The 2013 clinical guidelines for the diagnosis and treatment of HIV/AIDS in HIV-infected Koreans. Infect Chemother. 2013;45(4):455-461.

doi: 10.3947/ic.2013.45.4.455

23. Pluye P, Robert E, Cargo M, Bartlett G, Ocathain A, Griffiths F, et al. Proposal: a mixed methods appraisal tool for systematic mixed studies reviews. Montréal: McGill University; 2011. p. 1-8.

\section{Table}

\section{Table 1.}

Data extraction form

\begin{tabular}{|l|}
\hline Characteristics of the articles \\
\hline First author \\
Year of publication \\
Study design \\
Country \\
\hline Study population \\
\hline Outcomes \\
\hline Dependent variable \\
\hline Independent variable \\
\hline Barriers \\
\hline Facilitators \\
\hline
\end{tabular}


Figures

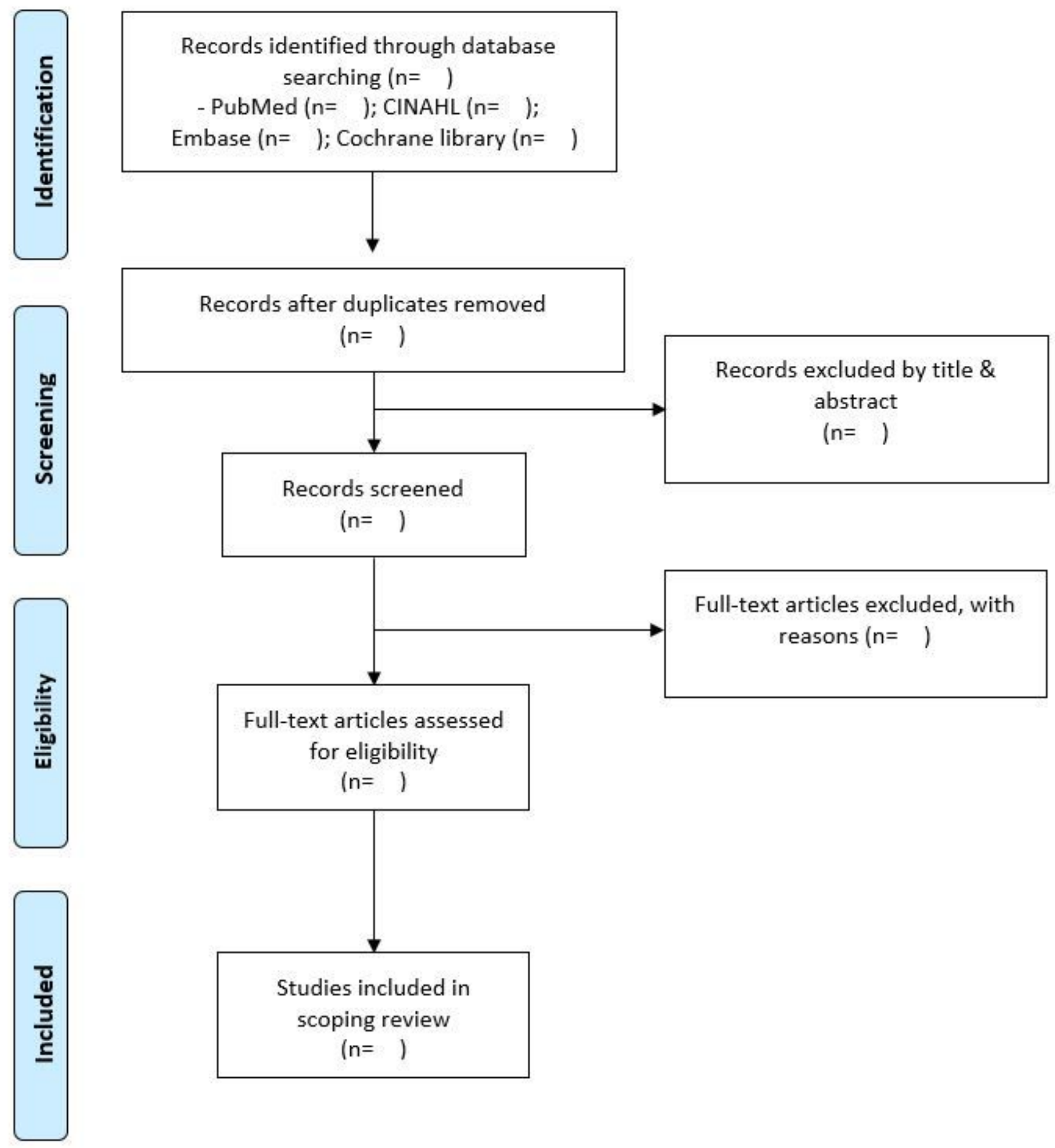

Figure 1

PRISMA flow diagram

Supplementary Files 
This is a list of supplementary files associated with this preprint. Click to download.

- Supplementalmaterial1.docx 